Archives de sciences sociales des religions

122 | avril - juin 2003

Varia

\title{
De la régulation étatique du religieux
}

Nathalie Luca

\section{OpenEdition}

Journals

Édition électronique

URL : http://journals.openedition.org/assr/1503

DOI : 10.4000/assr.1503

ISSN : $1777-5825$

Éditeur

Éditions de l'EHESS

Édition imprimée

Date de publication : 1 avril 2003

ISBN : 2-222-96732-5

ISSN : 0335-5985

Référence électronique

Nathalie Luca, «De la régulation étatique du religieux », Archives de sciences sociales des religions [En ligne], 122 | avril - juin 2003, mis en ligne le 10 novembre 2005, consulté le 19 avril 2019. URL : http:// journals.openedition.org/assr/1503 ; DOI : 10.4000/assr.1503

(C) Archives de sciences sociales des religions 


\title{
DE LA RÉGULATION ÉTATIQUE DU RELIGIEUX
}

\author{
À propos de : \\ Religions, droit et sociétés dans l'Europe \\ communautaire. Actes du XIII ${ }^{\mathrm{e}}$ colloque de l'Institut de \\ Droit et d'Histoire Religieux (IDHR), Aix-en-Provence \\ 19-20 mai 1999, Aix-en-Provence, Presses universitaires \\ d'Aix-Marseille, 2000, 294 p. \\ THIERSTEIN (Joel), KAMALIPOUR (Yahya R.), \\ eds., Religion, Law, and Freedom. A Global \\ Perspective, Westport (Conn.), Praeger, 2000, 242 p. \\ (index). \\ AHDAR (Rex J.), éd., Law and Religion, Dartmouth, \\ Hants (GB), Vermont (NA), Ashgate, 2000, 229 p., \\ (bibliogr., index).
}

Les trois ouvrages collectifs présentés ici sont traités sous l'angle d'une problématique commune : celle des modalités de la régulation du religieux par l'Etat, et indirectement, des conditions d'institutionnalisation des groupes religieux. Il s'agit d'approches sociologiques, historiques ou juridiques s'appuyant essentiellement sur des terrains occidentaux et largement centrées sur les pays qui garantissent constitutionnellement la liberté des croyances religieuses voire qui se fondent sur la séparation des Églises et de l'État. Constatant que des liens subsistent malgré tout entre certaines institutions religieuses et les différents États, et que la liberté n'est pas égale pour tous, les auteurs développent une réflexion sur la gestion de ce paradoxe, pointant une définition implicite du religieux acceptable pour l'État.

\section{Des limites à la liberté religieuse ?}

Le principe de liberté religieuse implique, idéalement, que l'État soit indifférent aux activités des communautés religieuses et des croyants. Il en va néanmoins 
autrement. À en croire les différents auteurs, l'État intervient dans ces domaines « de façon inimaginable il y a un siècle » (1). La liberté religieuse a déjà des limites directement posées dans les textes officiels, tant au niveau international, que sur le plan national. L'article 18 de la Déclaration universelle des Droits de l'Homme (1948), celui de la convention sur les droits civils et politiques (1966), l'article $1^{\text {er }}$ de la déclaration de 1981 sur l'élimination de toute forme d'intolérance et de discrimination basée sur une religion ou une croyance, ou encore l'article 9 de la Convention européenne des Droits de l'Homme, formulent cette limite par référence aux lois prescrites et nécessaires pour protéger l'ordre public, la santé et la morale ou les libertés fondamentales d'autrui. Au niveau national, les constitutions des pays européens reconnaissent la liberté religieuse tant que « les pratiques n'entrent pas en contradiction avec l'ordre public ou les bonnes mœurs » (Autriche, Danemark, Finlande), tant que «le rite ne contrarie pas la moralité » (Italie); ou tant que cette liberté n'enfreint pas «un dessein acceptable à une société démocratique » (Suède) (2).

De fait, la liberté religieuse n'est effective que pour l'individu : « elle implique un domaine de liberté pour les individus à adhérer à un ensemble de croyances et l'obligation des pouvoirs publics à ne pas s'en mêler» (3). Simultanément, elle n'existe que dans la sphère privée, «toute forme de manifestation [publique étant] potentiellement sujette à restriction », dès lors qu'elle « rentre en conflit avec une autre convention de droit »(4). Ainsi, la liberté de croire est certes personnelle et absolue, mais la liberté d'action ne peut l'être.

Plus subjectivement, cette liberté se heurte ensuite aux principes culturels de la morale dominante, principes qui rendent très élastique la notion même de restriction. Cette liberté est donc hautement problématique pour les États, les dimensions sociale, institutionnelle et communicationnelle de la religion la plaçant en permanence sur la scène publique. Par ailleurs, les contacts étroits que les gouvernements entretiennent avec les institutions de la société civile rendent difficilement opérationnel le concept d'une stricte séparation, et nuisent à toute scrupuleuse neutralité. Ce problème est aggravé par la très grande diversification actuelle des groupes religieux qui oblige les États à marquer leurs préférences pour telle ou telle institution religieuse. Ainsi, le christianisme qui, dans tout l'Occident, reste en position dominante, est-il privilégié tant sur le plan légal que sur le plan social. Sa centralité pénalise nécessairement les croyances minoritaires et pose une question de fond: est-il possible « d'adopter une attitude parfaitement non-discriminatoire entre plusieurs religions qui ont des divergences éthiques ?» (5).

(1) Rex J. Ahdar, ed., "The Inevitability of Law and Religion: An Introduction », in Law and Religion, Dartmouth, Hants (GB), Vermont (NA), Ashgate, 2000, p. 1.

(2) Manny PARAschos, "Religion, Religious Expression and the Law in the European Union », in Joel Thierstein, Yahya R. Kamalipour, eds, Religion, Law, and Freedom. A Global Perspective, Westport (Conn.), Praeger, 2000, pp. 20-22.

(3) Sophie C. van Bijsterveld, «Religion, Law and Policy in the Wider European Arena: New Dimensions and Developments », in Rex J. ADHAR, ed., op. cit., p. 175.

(4) Malcom D. Evans, "The United Nations and Freedom of Religion: The Work of the Human Rights Commitee », in Rex J. AhDar, ed, op. cit., p. 48.

(5) Julian Rivers, « From Toleration to Pluralism: Religious Liberty and Religious Establishment under the United Kingdom's Human Rights Act », in Rex J. AdHAR, ed., op. cit., p. 134. 
Leur sélection implicite repose avant tout sur le respect des critères occidentaux des droits de l'homme. Cependant, ces critères sont perçus par les groupes religieux refoulés comme émanant d'un "impérialisme occidental des droits humains » qui favorise l'expansion du christianisme dans le reste du monde, et freine l'intégration des religions nouvelles en Occident dès lors que leurs référents culturels exotiques n'adhèrent pas à l'éthique occidentale (6). On comprend néanmoins la perplexité des instances nationales ou internationales lorsqu'elles doivent traiter de la légitimité de la liberté religieuse dans le cas de groupes, qui, entre autres exemples extrêmes, " cultivent, possèdent, distribuent et adorent », pour raison religieuse, la marijuana (7) - cas de l'Assemblée de l'Église de l'Univers, d'origine indienne, présente sur le sol canadien. Les conflits entre le droit et les lois sont bien au cœur de la polémique. La liberté religieuse est contrainte par des lois directement influencées par la culture dominante.

\section{Des critères intangibles ?}

Quels sont donc les critères sur lesquels ne transige pas un État ? Cette question permet fort utilement de rappeler que le Nouveau Monde s'interroge tout autant que l'Ancien sur les limites de l'expression religieuse. La politique étatsunienne varie face aux particularités de certaines pratiques religieuses selon les courants et les époques. Le premier amendement n'est ni simple à comprendre, ni simple à appliquer. Au départ, il ne valait que pour le gouvernement fédéral, et n'interdisait pas aux États d'établir une religion (8). Depuis, le « mur de séparation entre l'Église et l'État » est comparé soit à de la pâte à modeler, soit à du ciment, soit à un matériau à consistance variable. On note ainsi l' "idéal séparationiste » - peu plaidé -, l'«idéal de neutralité », et l'«idéal de la liberté religieuse ». La Cour Suprême choisit essentiellement entre les deux dernières options, aidant la religion quand elle protège sa liberté, et limitant cette dernière quand elle plie la religion aux lois communément appliquées. Les points de vues changent selon que l'on se place du côté des intérêts des religions majoritaires ou de celui des religions minoritaires. Mais ce qui prime en réalité, c'est la gestion que l'on entend avoir de la vie publique des religions. «Il est clair (...) qu'une stricte séparation implique un traitement spécial de la religion, ce que la neutralité interdit. » (9) De fait, ici comme ailleurs, les cours de justice font un travail d' "institution normative », « renforçant les normes, les croyances et les pratiques considérées comme importantes dans une société donnée ». Cela les amène à opérer "au détriment des groupes religieux impopulaires et dont les pratiques sont préjugées répréhensibles » (10).

(6) Préface de Cees J. Hamelink, in Joel Thierstein et Yahya R. Kamalipour, eds., op. cit.

(7) Malcom D. Evans, op. cit., p. 43.

(8) Ron Manuto, «Historical Perspectives on Contemporary Freedom in America », in Joel Thierstein et Yahya R. KAMALIPOUR, eds., op. cit, p. 8.

(9) Michael W. McConnel, « Neutrality, Separation and Accommodation: Tensions in American First Amendement Doctrine », in Rex J. AdHAR, ed., op. cit., p. 64.

(10) James T. RichaRdSON, « Discretion and Discrimination in Legal Cases involving Controversial Religious Groups and Allegations of Ritual Abuse », in Rex J. AdHAR, ed., op. cit., pp. 111-112. 
Des deux côtés de l'Océan, on saisit ainsi l'importance prêtée à la compatibilité du groupe religieux avec les idéaux nationaux. Aux États-Unis, les Mormons ont été violemment persécutés par le gouvernement, avec l'aval de la justice, pour leur polygamie, caractérisée comme « une pratique barbare violant la loi naturelle et attaquant les fondements moraux d'une société civilisée » (11). Plus récemment, continue Marie A. Failinger, « les Indiens américains qui rejettent le point de vue moral selon lequel les êtres humains sont les maîtres d'un monde conçu pour leur propre bénéfice provoquent un clash constitutionnel majeur dans une société fondée sur le droit à la propriété ». La même observation vaut pour les pacifistes absolus qui sont en rupture avec une « nation qui se perçoit comme le policier du monde » (12).

Sur le vieux continent, la notion de compatibilité est tout aussi importante. L'exemple de l'islam que présente Franck Frégosi permet assez bien d'en tracer les contours. L'Europe compte entre 9 à 14 millions de musulmans dont il faut réussir l'intégration. Une réflexion s'est donc imposée. Elle a révélé que l'institutionnalisation d'un groupe religieux nécessite de la part des pratiquants qu'ils reconnaissent la primauté de leur nouveau lieu de résidence sur celui qu'ils ont quitté. Elle implique qu'ils se plient à la législation territoriale lorsque celle-ci est en désaccord avec la loi religieuse - éventuellement en application dans leur pays d'origine. Un détachement des racines s'impose donc pour faciliter un nouvel enracinement dans la culture du pays d'accueil. Les pays d'origine des immigrants ne doivent pouvoir exercer un droit de regard sur les populations émigrées. Ainsi l'institutionnalisation de l'islam suppose-t-elle l'émergence d'un « islam autochtone », européen. Ce dernier officialise « le caractère plus ou moins achevé, durable, pérenne de son insertion ». Son adaptation lui permet dès lors de participer " au système politique dominant, en d'autres termes, [elle autorise] sa normalisation sociale » (13). Pour Franck Frégosi, cette normalisation nécessite que se développent des lieux de formation de l'islam, subventionnés par l'État, dans l'espace européen. C'est dans ces lieux qu'un islam typiquement européen pourrait réellement émerger et, dès lors, dialoguer avec le système politique dominant.

\section{Conséquences économiques de la régulation étatique}

Ce dernier constat montre l'importance de la dimension économique dans l'appréciation des types de relation qu'un État instaure avec les groupes religieux qui se développent sur son territoire : bénéficient-t-ils ou non de l'exonération de certaines de leurs charges? Obtiennent-ils ou non des subventions de l'État? L'application d'une stricte séparation des Églises et de l'État devrait-elle se

(11) Marie A. FAilinger, « Wondering after Babel: Power, Freedom and Ideology in US Supreme Court Interpenetrations of the Religion Clauses », in Rex J. ADHAR, op. cit., p. 97.

(12) Idem.

(13) Franck FrÉGOSI, « L'islam en Europe, entre dynamiques d'institutionnalisation, de reconnaissance et difficultés objectives d'organisation ", in Religions, droit et sociétés dans l'Europe communautaire, Actes du XIII e colloque de 1'Institut de Droit et d'Histoire Religieux (IDHR), Aix-en-Provence 19-20 mai 1999, Aix-en-Provence, Presses universitaires d'Aix-Marseille, 2000, p. 95. 
traduire par l'absence de rapport financier entre les deux, ce qui n'est observé nulle part, alors même que le financement public d'une institution religieuse peut, aussi, être un moyen de soumettre la religion aux normes étatiques ? Historiquement, rappelle Lionel Panafit en s'attachant au cas français, «c'est de manière concomitante au processus de sécularisation de l'espace public que fut instaurée (la) rémunération administrative des ministres du culte »(14). La différenciation de l'Église et de l'État a placé l'État au-dessus de l'Église. La prise en charge économique de l'Église la fonctionnarisait, et, par là même, la soumettait. On comprend dès lors que ce soit « au sein même de l'Église catholique qu'il (ait été) possible de trouver les premiers opposants à cette rémunération » (15). Or, c'est précisément en ne salariant, ni ne subventionnant aucun culte que la loi de 1905 est censée instaurer la laïcité. Comme la plupart des législations européennes, la France accorde néanmoins «des libéralités financières ou fiscales pour certains cultes, et par cette entremise établit, de fait, des reconnaissances explicites ou non de ceux-ci » (16).

Lionel Panafit constate en effet, avec Francis Messner, que dans toute l'Europe occidentale, la reconnaissance des cultes se fait par un système de défiscalisation, de financement d'aumôneries ou d'établissements scolaires confessionnels, d'entretien des édifices religieux, d'émissions religieuses sur les chaînes publiques de la télévision voire, dans certains pays, de reconnaissance des effets civils des mariages religieux. Or, ne sont défiscalisés que les groupes dont l'État reconnaît la dimension sociale, c'est-à-dire, finalement, la compatibilité de leurs valeurs avec celles de la société. Ainsi l'institutionnalisation d'un groupe religieux passe par sa visibilité sociale, visibilité qui lui permet de participer au système politique dominant, et qui est reconnue, officialisée par une relation économique qui se met en place entre lui et l'État. Ce système rapproche les cultes des associations d'utilité publique (17).

\section{La dimension sociale des cultes}

Les paradoxes entre la liberté de croire et l'encadrement législatif rigoureux de la pratique cultuelle, entre le «mur de séparation » américain - ou la laïcité française - et le système de "préférences » implicite des cultes, font partie des surprises de la modernité. On comptait pouvoir normer ou souder la société en dehors d'un socle religieux rigide, et l'on se retrouve face à une société sans socle : l'individualisme a été intronisé pulvérisant l'être-en-commun, et délaissant le collectif. La politique se retrouve face à un problème majeur inattendu : redonner consistance au bien commun. Pour y parvenir, toutes les ressources disponibles sont mobilisées, favorisant l'émergence de nouveaux liens avec les religions tradition-

(14) Lionel PANAFIT, «Les relations religions-État en Europe au prisme de l'économique », in Religions, droit et société dans l'Europe communautaire, op. cit., p. 51.

(15) Idem.

(16) Idem, p. 53

(17) Cette comparaison est faite par Francis Messner, « Les relations États-religions dans les pays membres de l'Union européenne », in Religions, droit et société dans l'Europe communautaire, op. cit., p. 35. 
nelles porteuses de sens (18). De fait, constate Jean Chelini, si un temps, l'Église catholique a pu se marginaliser, elle a retrouvé, depuis le début du règne de JeanPaul II, son rôle social public. «L'Église universelle, les Églises nationales peuvent et doivent se prononcer sur les questions morales : sur la famille, sur le rejet de l'avortement et de la contraception, de l'euthanasie, et le faire de manière universelle (...). L’Église doit non seulement déclarer mais intervenir sur le terrain des nations » (19).

Un autre paradoxe réside cependant dans le fait que les religions qui se déprivatisent avec le plus de hardiesse le font essentiellement pour participer à la contestation. Pour R. J. A., c'est le cas des religions considérées comme « réactionnaires », comme « traditionalistes » ou comme «fondamentalistes » qui se revitalisent aujourd'hui : "la résurgence de la plupart des religions du monde dans une forme conservatrice voire militante a été une des réponses à la sécularisation » (20). Du coup une nouvelle question est posée à laquelle les pays répondent diversement selon les rapports établis entre les religions et l'État : « Des individus ou des institutions religieuses peuvent-ils exercer une influence politique basée sur leurs fondements théologiques » (21) ? Certains apportent une réponse négative à cette question. D'autres estiment que ces institutions sont représentatives d'une partie de la population et, qu'à ce titre, leur influence politique est légitime, ils répondent donc positivement. D'autres enfin reconnaissent la possibilité d'une utilisation éthique d'arguments religieux. Ces positions varient néanmoins, à nouveau, en fonction de la compatibilité de l'idéologie du groupe religieux avec celle de la société environnante. Plus l'enracinement religieux et culturel est profond, plus les frontières de la compatibilité risquent d'être clairement posées. Il faudrait ajouter, semble-t-il, au constat de Marie A. Failinger, que plus la mémoire du patrimoine est flottante et la menace de l'oubli forte, plus le rappel de ces frontières risque d'être fortement mobilisé, comme un dernier sursaut pour la sauvegarde de l'identité. La nature pluriculturelle des États-Unis les place nécessairement dans une situation très différente d'une France culturellement marquée par le seul catholicisme. Il n'en demeure pas moins que les États-Unis « ont une culture morale identifiable, basée principalement sur le protestantisme»(22) et que cette culture marque clairement leurs choix.

C'est peut-être au Royaume-Uni que se dégage le plus nettement une volonté de faire face à la pluralité religieuse et à la diversité des fondements éthiques qui l'accompagne. La tournure plus libérale qu'y prend la démocratie depuis 1995 a amené les responsables politiques à voter en 1998 une Déclaration des Droits de l'Homme (the Human Rights Act) qui incorpore la convention européenne aux lois domestiques. Elle reconnaît la nature publique des faits religieux et présente une stratégie apte à réguler les conflits qui en découlent. Il s'agit d'identifier une sphère d'action protégée contre toute contestation émanant du monde séculier, du gouvernement ou d'autres acteurs religieux. Une série de «mini-institutions » distinctes

(18) Théorie développée par Marcel Gauchet, dans La religion dans la démocratie. Parcours de la laïcité, Gallimard, Paris, 1998 (coll. « Le débat »).

(19) Jean Chelini, «La position actuelle de l'Église catholique sur la place de la religion dans la société », in Religions, droit et société dans l'Europe communautaire, op. cit., p. 77.

(20) Rex J. AHDAR, op. cit., p. 4.

(21) Marie A. Failinger, op. cit., p. 90.

(22) Idem, p. 95. 
religieusement et éthiquement garantirait ainsi aux groupes religieux un niveau d'autonomie et d'autorité protégées. Reste à savoir jusqu'où la mise en place de ce pluralisme religieux " pourra se poursuivre sans se heurter à la place symbolique $\mathrm{du}$ christianisme toujours implicite dans les arrangements constitutionnels du Royaume Uni » (23).

La faisabilité d'une réelle acceptation de la pluralité religieuse reste hypothéquée par la difficulté pour un État d'être indifférent à la nature du rôle social des religions. Celles-ci l'intéressent davantage lorsqu'il leur reconnaît la capacité d'apporter un soutien moral, éthique dans une société en perte de repères, ou encore, lorsque leur participation à la fabrique de la société lui apparaît indéniable. C'est certainement ce dont a pris acte le colloque sur « l'enseignement du fait religieux à l'école " organisé à Paris par le Ministère de l'éducation nationale en novembre 2002. Il a mis à jour, de façon explicite, le besoin qui s'exprime, au cœur même de la laïcité, de se réapproprier l'héritage culturel des religions. Aux ÉtatsUnis, les enjeux de cet héritage ont une fonction politique à laquelle le gouvernement recourt en cas de crise. Pour les traditionalistes américains au pouvoir, une culture non religieuse est perçue comme une menace : la guerre froide a ainsi fait de l'ennemi celui qui ne croyait pas en Dieu. L'ennemi s'est certes déplacé aujourd'hui mais il renforce l'importance accordée aux institutions religieuses dans la vie des Américains, à tel point qu'il réactualise le débat sur la possibilité d'enseigner à l'école publique les valeurs morales chrétiennes. Pour les libéraux, l'Église et l'État représentant ensemble une menace pour la liberté de conscience individuelle, seule une morale laïque peut être enseignée à l'école. Mais il existe aussi une approche plus contextuelle et pragmatique qui ne veut ni transformer Noël en simple solstice d'hiver - ce que souhaitent les libéraux - ni remettre le catéchisme à l'ordre du jour - secret espoir des traditionalistes -, mais enseigner les religions dans toute leur diversité (24).

On le voit, nulle part la question de la visibilité sociale des groupes religieux ne relève que de la stricte compétence juridique. Elle est régulée par des choix de politiques publiques plus ou moins explicités, plus ou moins libéraux ou conservateurs. Ils révèlent la difficulté de gérer sur le terrain national les conséquences concrètes de la mondialisation culturelle et de la multiplication des éthiques incompatibles entre elles qu'elle génère. Certes on peut chercher à circonscrire ces problèmes à l'intérieur d'une politique antisectes dont la vocation pour l'État est finalement de poser les limites de ce qui est socialement acceptable pour une société donnée. On peut, au contraire, chercher à faire exploser ces limites par un système de protection des variétés éthiques. Mais quelles que soient les solutions retenues, elles obligent les États à se repositionner face à la culture religieuse dominante. La mondialisation se heurte alors au désir de maintenir son «authenticité » et sa particularité, désir peut-être d'autant plus développé que cette « authenticité » devient évanescente. Cela est peu fait pour avaliser l'absolue diversité, ni l'absolu relativisme.

Nathalie LUCA

Centre d'Études Interdisciplinaires des Faits Religieux CNRS

(23) Julia Rivers, op. cit., p. 154.

(24) Marie A. FAILINGER, op. cit., pp. 94-97. 
